\title{
Clinical Efficacy of Telemedicine Compared to Face-to-Face Clinic Visits for Smoking Cessation: Multicenter Open-Label Randomized Controlled Noninferiority Trial
}

Akihiro Nomura $^{1,2,3}$, MD, PhD; Tomoyuki Tanigawa ${ }^{1,4}$, MD; Tomoyasu Muto ${ }^{5}$, MSc; Takafumi Oga ${ }^{6}$, MD; Yasushi Fukushima $^{7}$, MD; Arihiro Kiyosue ${ }^{8}$, MD; Masaki Miyazaki ${ }^{9}$, MD; Eisuke Hida ${ }^{10}$, PhD; Kohta Satake ${ }^{1,5}$, MPH, MBA, MD

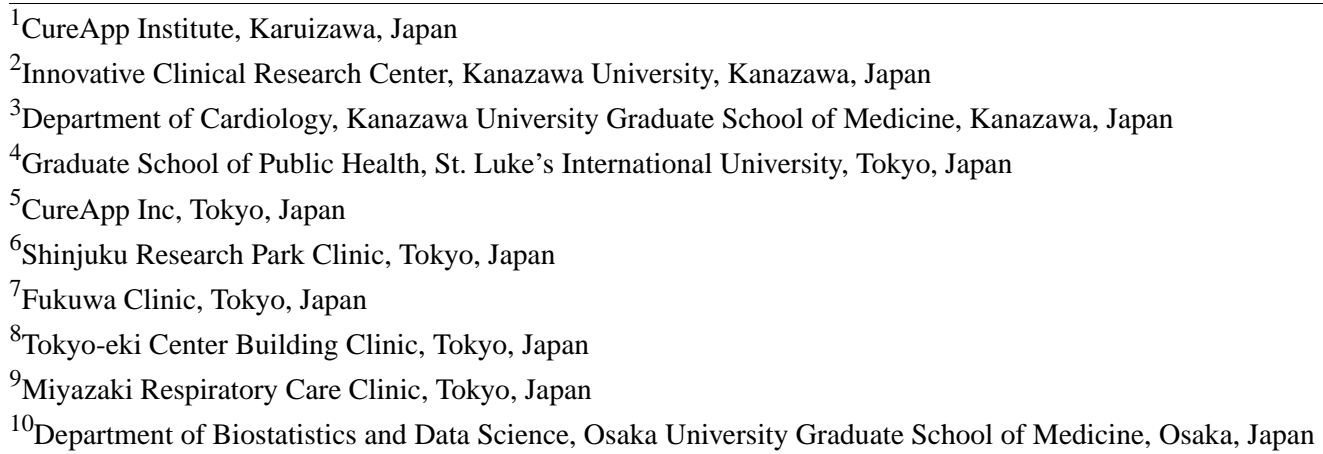

\section{Corresponding Author:}

Akihiro Nomura, MD, PhD

Innovative Clinical Research Center

Kanazawa University

13-1 Takaramachi

Kanazawa, 920-8641

Japan

Phone: 81762652049

Email: anomura@med.kanazawa-u.ac.jp

\section{Abstract}

Background: Tobacco is a major public health concern. A 12-week standard smoking cessation program is available in Japan; however, it requires face-to-face clinic visits, which has been one of the key obstacles to completing the program, leading to a low smoking cessation success rate. Telemedicine using internet-based video counseling instead of regular clinic visits could address this obstacle.

Objective: This study aimed to evaluate the efficacy and feasibility of an internet-based remote smoking cessation support program compared with the standard face-to-face clinical visit program among patients with nicotine dependence.

Methods: This study was a randomized, controlled, open-label, multicenter, noninferiority trial. We recruited nicotine-dependent adults from March to June 2018. Participants randomized to the telemedicine arm received internet-based video counseling, whereas control participants received standard face-to-face clinic visits at each time point in the smoking cessation program. Both arms received a CureApp Smoking Cessation smartphone app with a mobile exhaled carbon monoxide checker. The primary outcome was a continuous abstinence rate (CAR) from weeks 9 to 12 . Full analysis set was used for data analysis.

Results: We randomized 115 participants with nicotine dependence: 58 were allocated to the telemedicine (internet-based video counseling) arm and 57, to the control (standard face-to-face clinical visit) arm. We analyzed all 115 participants for the primary outcome. Both telemedicine and control groups had similar CARs from weeks 9 to 12 (81.0\% vs 78.9\%; absolute difference, $2.1 \% ; 95 \% \mathrm{CI}-12.8$ to 17.0$)$, and the lower limit of the difference between groups $(-12.8 \%)$ was greater than the prespecified limit $(-15 \%)$.

Conclusions: The application of telemedicine using internet-based video counseling as a smoking cessation program had a similar CAR from weeks 9 to 12 as that of the standard face-to-face clinical visit program. The efficacy of the telemedicine-based smoking cessation program was not inferior to that of the standard visit-based smoking cessation program. 
Trial Registration: University Hospital Medical Information Network Clinical Trials Registry: UMIN000031620; https://upload.umin.ac.jp/cgi-open-bin/ctr_e/ctr_view.cgi?recptno=R000035975.

(J Med Internet Res 2019;21(4):e13520) doi: 10.2196/13520

\section{KEYWORDS}

smoking cessation; nicotine dependence; telecare; telemedicine; mHealth; digital therapeutics; mobile phone

\section{Introduction}

Tobacco is a major public health concern and the biggest preventable cause of a variety of disorders such as cerebro- and cardiovascular diseases, malignant tumors, and chronic obstructive pulmonary disease [1,2]. In Japan, the estimated number of smokers is more than 20 million, and smoking is responsible for approximately 130,000 deaths per year [3]. Thus, reducing the prevalence of smoking would help prevent deaths from life-threatening diseases [4].

To help quit smoking, Japan provides a smoking cessation program for patients with nicotine dependence. This 12-week program mainly consists of face-to-face clinic visits, involving counseling with a primary physician, checking exhaled carbon monoxide (CO) concentration, and prescribing smoking cessation medications [5]. Face-to-face clinic visits enable physicians to directly perform counseling, physical examinations, and various tests in person. However, despite these intensive efforts to have patients complete the program, more than half of the program participants could not complete the entire program [6]. The majority of patients with nicotine dependence in Japan are typically employed men who are extremely busy and unwilling to spend a large portion of their day visiting the clinic to receive the smoking cessation program [6]. Program dropout is normally considered equivalent to smoking cessation failure [7]. Therefore, promoting dedication and completion of the smoking cessation program could be crucial to making these people succeed at quitting smoking.

Recently, telemedicine, defined as remote delivery of health care via the internet, was considered one of the useful methods for providing medical care to patients $[8,9]$. Telemedicine minimizes patients' burden of visiting a health institution and waiting for consultations with their physicians. Telemedicine could also be suitable for delivering a smoking cessation support program. Considering the preliminary report that $75 \%$ of participants could complete the smoking cessation program when conducted via telemedicine, the requirement of regular face-to-face visits at a clinic might be the reason for the low completion rate of the smoking cessation program [10]. Therefore, telemedicine that enables the smoking cessation program participants to receive their regular counseling via the internet could have the potential to improve the overall smoking cessation success rate among patients with nicotine dependence by providing them easier access to the program. However, it remains uncertain whether telemedicine using an internet-based video counseling system is effective for delivering the smoking cessation program compared to the standard face-to-face clinical visits.
In this study, we tested the clinical efficacy and feasibility of telemedicine using internet-based Web counseling compared to a standard face-to-face clinical visit in the smoking cessation program among patients with nicotine dependence.

\section{Methods}

\section{Trial Design and Participants}

This trial was a randomized, controlled, open-label, multicenter, noninferiority trial. Details of the trial protocol have been described elsewhere [11]. In brief, participants in both arms underwent the smoking cessation program used in Japan [5]. For the telemedicine arm, the entire smoking cessation program was conducted remotely via an internet-based video counseling system, except for the first registration visit [12]. For the control arm, participants followed the standard smoking cessation program conducted through face-to-face clinic visits. Participants in both arms used the CureApp Smoking Cessation (CASC) system [13]. The primary outcome was a biochemically validated continuous abstinence rate (CAR) from weeks 9-12.

We recruited individuals with nicotine dependence from March to June 2018. We conducted follow-up for 24 weeks. Only the participants who met all the inclusion criteria were included; those who met any of the exclusion criteria were excluded [11]. Briefly, we included participants who were diagnosed with nicotine dependence (Tobacco Dependence Screener score $\geq 5$ points) [14], had a Brinkman index $\geq 200$, had the will to quit smoking immediately, agreed to undergo the smoking cessation program, and could use a smartphone. We excluded participants who had severe mental illness, could not tolerate the follow-up for 6 months, had used smoking cessation supplements or medication before the registration, planned to use any smoking cessation aids or to participate in any kind of smoking, or had regular clinic visits for diseases other than nicotine dependence planned within 12 weeks of registration.

Primary physicians at each clinic obtained written informed consent from all trial participants. We confirmed that clinics participating in this trial could provide the standard smoking cessation support program and had the necessary equipment to provide Web-based telemedicine (eg, $\mathrm{WiFi}$ access in the facility). We conducted this trial in compliance with the Declaration of Helsinki, Medical Device Good Clinical Practice guidelines, and all other applicable laws and guidelines in Japan. The trial protocol was approved by the Tokyo-Eki Center-Building Clinic institutional review board. We reported the trial according to CONSORT-EHEALTH (V 1.6.1). This trial was registered at the University Hospital Medical Information Network Clinical Trials Registry (UMIN000031620). 


\section{Randomization}

We used the stratified-block randomization (four blocks) method with a 1:1 allocation ratio to achieve equal assignment to two arms with stratification of the trial sites. Participants were allocated to either the telemedicine arm or the control arm. The randomization was performed by the staff at each participating clinic at the time of participants' registration, using a computer-generated random sequence.

\section{Procedures}

Participants allocated to the telemedicine arm received internet-based Web counseling for the smoking cessation program. Participants assigned to the control arm received the conventional face-to-face clinic visits for the smoking cessation program. Both arms also received the CASC smartphone app and a mobile exhaled $\mathrm{CO}$ checker during the trial period (24 weeks).

The standard smoking cessation program in Japan consists of five face-to-face clinic visits lasting for 12 weeks, including doctor consultations and exhaled $\mathrm{CO}$ checks at a registered institution or clinic [5]. All study participants visited their primary physicians at their first visit to confirm that they fully understood the trial protocol. At this visit, the physicians decided to prescribe appropriate smoking cessation medication, to provide guidance in accordance with the standard program procedure, and to provide participants with the CASC smartphone app integrated with a mobile $\mathrm{CO}$ checker.

Following the first visit, telemedicine participants were supposed to receive counseling via the internet-based video counseling system with a standardized telemedicine platform application [12] instead of visiting their clinics to see their primary physicians. As in the standard program, they met with their physicians via video counseling at each planned visit at weeks $2,4,8,12$, and 24 . The control participants were supposed to visit their clinics at weeks $2,4,8,12$, and 24 .

\section{Overview of CureApp Smoking Cessation}

The CASC system was developed by CureApp, Inc. (Tokyo, Japan). Details of the system have been demonstrated elsewhere [13]. The CASC system consists of the CASC smartphone app [15], mobile exhaled CO checker, and Web-based personal computer for primary physicians. For the telemedicine arm, the primary physicians provided the app prescription code to the participants at their first visit in the outpatient clinics. The telemedicine participants downloaded the app through their smartphones; activated the app by entering the code; and keyed in their baseline data, motivation, and self-confidence regarding smoking cessation. The CASC smartphone app has four main components: (1) keeping a smoking cessation digital diary (filled in once a day), (2) lectures and educational videos helping its users to quit smoking, (3) interactive counseling by chat-bot, and (4) daily measurement and recording of exhaled $\mathrm{CO}$ concentration levels at home using the mobile $\mathrm{CO}$ checker. The Web-based personal computer software for the primary physicians provided a data-management app from patients' CASC smartphone apps and advice for physicians to follow the national clinical guidelines.

\section{Outcomes}

The primary outcome was the biochemically validated CAR from weeks 9-12, which was consistent with the previous clinical trial of varenicline in Japan [16]. This measure is defined as the percentage of individuals continuously not smoking (success) during the specified period. This study defined smoking cessation success as self-reported continuous abstinence as well as exhaled $\mathrm{CO}$ concentration $\leq 10 \mathrm{ppm}$ during the given period [16]. For example, CAR from weeks 9-12 indicates that the patient achieved smoking cessation success if he/she self-reported continuous abstinence for 9-12 weeks and his/her exhaled CO concentration was $\leq 10 \mathrm{ppm}$ at week 12. We also evaluated the following secondary outcomes: CAR from weeks 9-24; changes in the scores on the Mood and Physical Symptoms Scale (MPSS) [17] and the 12-item French version of the Tobacco Craving Questionnaire (FTCQ-12) [18]; the Kano Test for Social Nicotine Dependence (KTSND) score [19] at weeks 8, 12, and 24; Nicotine Dependence Cognition Scale (NDCS) score at weeks 12 and 24 [11]; and all adverse events during the trial.

\section{Sample Size and Inferiority Margin}

From previous pilot studies of a CASC smartphone app, the difference in CAR from 9-12 weeks between the CASC smartphone app group (78\%) and historical control groups (not using the app; $54 \%$ ) is $24 \%$ [20-22]. Therefore, we hypothesized that the telemedicine group would not provide clinically worse CAR from 9-12 weeks compared to the control group (prespecified margin of $15 \%$ based on estimated $80 \%$ CARs in both groups) [11]. We calculated the required sample size as 114 (57 per each arm) based on the precision of estimate that the lower limit of the $95 \%$ CI of the difference between treatment effects exceeded the threshold amount of $15 \%$. Therefore, we aimed to recruit at least 114 participants to allow for this sample size.

\section{Statistical Analysis}

We compared all endpoints between the telemedicine and control groups. Baseline characteristics were described by means and SDs, medians and interquartile ranges (for continuous variables), or proportions (for categorical variables). We analyzed the primary outcome using the full analysis set (excluding participants who violated the inclusion or exclusion criteria). In case a patient discontinued an allocated treatment, the case was considered as smoking cessation failure. We compared CARs between telemedicine and the control groups using a logistic regression model with crude odds ratios (ORs). For all outcomes, summary statistics and group difference measures (eg, ORs by logistic regression or mean differences) were presented with $95 \%$ CIs. We also tested if the CAR from 9-12 weeks of telemedicine and the control condition varied by subgroups. We assessed interactions of CAR from 9-12 weeks with each variable (greater than vs less than the median for continuous variables) and calculated ORs with $95 \%$ CIs in each subgroup. We used the Wilcoxon signed-rank test for comparing the scores regarding nicotine dependence between baseline and at weeks 12 and 24 in each group. R version 3.4.1 (R Foundation for Statistical Computing, Vienna, Austria) was used for all the analyses. 


\section{Results}

We randomized 115 participants to the telemedicine arm or control arm (Figure 1). Each arm was well balanced in the baseline characteristics (Table 1). All participants were prescribed smoking cessation medication at registration: varenicline for $55 \%$ and the nicotine patch for $45 \%$ of the participants. During the trial period, three participants discontinued their allocated treatment, two participants by consent withdrawal and one participant by loss to follow-up. Finally, all 115 participants were enrolled for further analyses.

Biochemically validated CARs from weeks 9-12 were $81.0 \%$ (95\% CI 71-91) in the telemedicine group and $78.9 \%$ (95\% CI 68-89) in the control group (Table 2). The absolute difference was $2.1 \%$ (95\% CI -12.8 to 17.0 ); the lower limit of the $95 \%$ CI $(-12.8 \%)$ was greater than the prespecified limit of $-15 \%$ (Multimedia Appendix 1). The OR was 1.14 (95\% CI 0.45-2.88). Moreover, CARs from weeks 9-24 were $74.1 \%$ (95\% CI 63-85) in the telemedicine group and $71.9 \%$ (95\% CI 60-84) in the control group. Therefore, there were no statistically significant differences between the telemedicine and control groups in CARs. In terms of program feasibility, adherence rates by session were both high (over 95\%) during the trial (Multimedia Appendix 1). In addition, there were no serious or device-related adverse events in the groups during the trial (Multimedia Appendix 1). CASC product issues during the trial were reported by four participants $(3.5 \%)$ : two issues were related to mobile $\mathrm{CO}$ checker connection failure (one in telemedicine group and one in control group), one was related to login failure in the control group, and one was related to other malfunction of the primary physician interface (failure of a chart closure) in the telemedicine group.

Next, we demonstrated the efficacy of the telemedicine on CAR from weeks 9-12 by subgroup. Although the KTSND had a moderate interaction with the outcome, we found little evidence of significant interactions for any of the subgroup analyses (Multimedia Appendix 1).

We also assessed the evolution of scores by MPSS, FTCQ-12, KTSND, and NDCS (Multimedia Appendix 1). During the 24-week trial period, all FTCQ-12, KTSND, and NDCS scores were significantly decreased over time in both groups. MPSS scores, especially regarding urges ("time spent with urges" and "strength of urges"), were also significantly reduced over time in both groups.

Figure 1. Trial flowchart. CASC: CureApp Smoking Cessation; SC: smoking cessation.

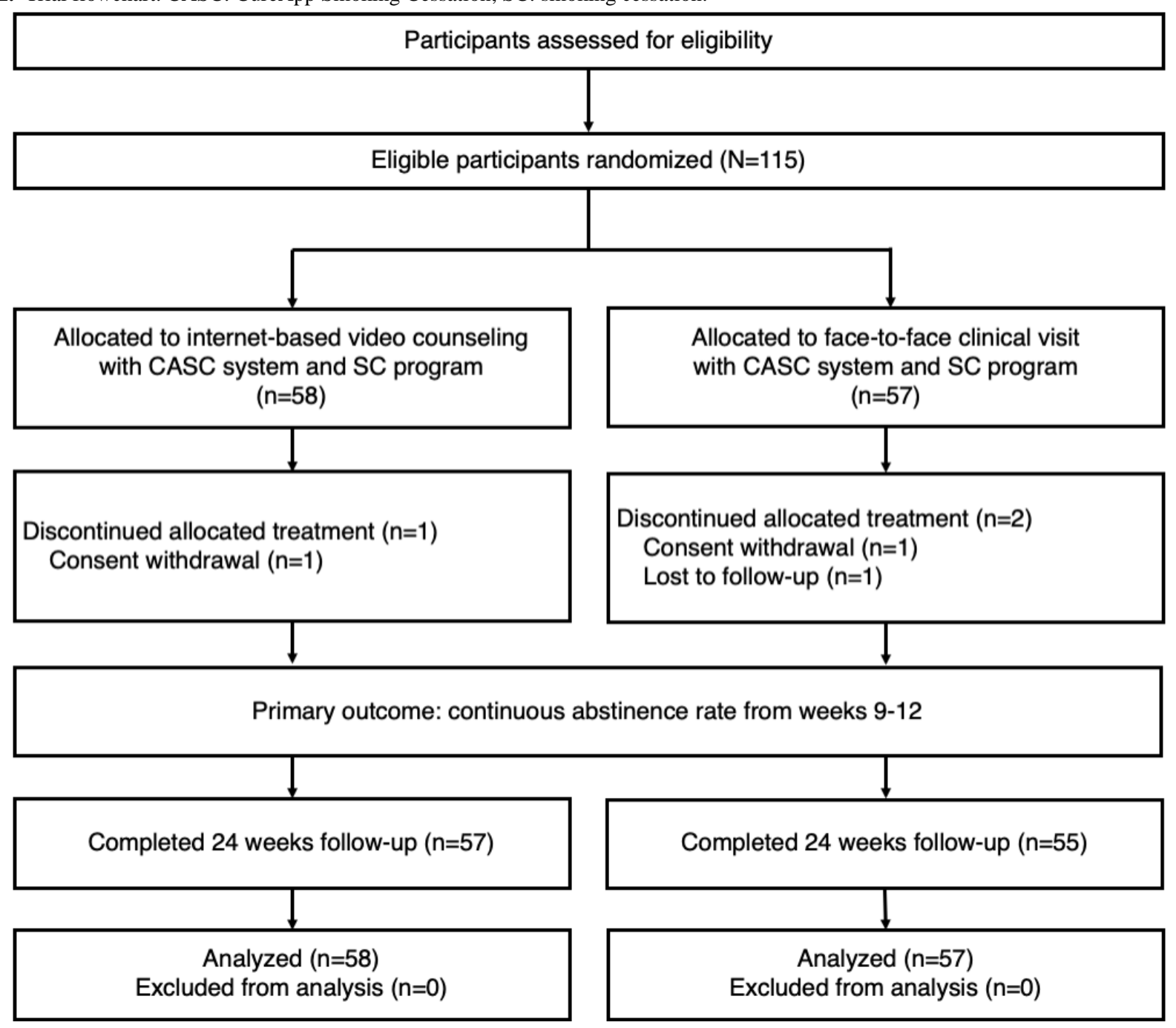


Table 1. Baseline characteristics of the trial participants.

\begin{tabular}{|c|c|c|c|}
\hline Characteristic & Total $(\mathrm{N}=115)$ & Telemedicine $(\mathrm{N}=58)$ & Standard care $(\mathrm{N}=57)$ \\
\hline Age (years), mean (SD) & $55(11)$ & $55(12)$ & $53(10)$ \\
\hline Male sex, n (\%) & $93(81)$ & $45(78)$ & $48(84 \%)$ \\
\hline Body mass index $\left(\mathrm{kg} / \mathrm{m}^{2}\right)$, median (interquartile range) & $23(21-26)$ & $23(21-26)$ & $23(21-25)$ \\
\hline Brinkman index, median (interquartile range) & $480(325-720)$ & $500(340-755)$ & $450(310-720)$ \\
\hline Cigarettes per day & $15(13-20)$ & $16(15-20)$ & $15(12-20)$ \\
\hline Years of smoking & $34(27-40)$ & $35(29-41)$ & $33(26-38)$ \\
\hline $\begin{array}{l}\text { Number of attempts of smoking cessation before the trial, } \\
\text { median (interquartile range) }\end{array}$ & $1(0-2)$ & $1(0-2)$ & $0(0-2)$ \\
\hline $\mathrm{TDS}^{\mathrm{a}}$ score, median (interquartile range) & $7(6-8)$ & $7(6-8)$ & $7(6-8)$ \\
\hline FTND $^{\mathrm{b}}$ score, median (interquartile range) & $5(3-7)$ & $5(4-7)$ & $5(3-6)$ \\
\hline KTSND $^{c}$ score, median (interquartile range) & $17(16-20)$ & $17(16-20)$ & $18(15-21)$ \\
\hline \multicolumn{4}{|l|}{ Comorbidities, n (\%) } \\
\hline Hypertension & $33(29)$ & $16(28)$ & $17(30)$ \\
\hline Diabetes mellitus & $9(8)$ & $5(9)$ & $4(7)$ \\
\hline Dyslipidemia & $42(37)$ & $23(40)$ & $19(33)$ \\
\hline \multicolumn{4}{|l|}{ Medication, $\mathrm{n}(\%)$} \\
\hline Varenicline & $63(55)$ & $29(50)$ & $34(60)$ \\
\hline Nicotine patch & $52(45)$ & $29(50)$ & $23(40)$ \\
\hline
\end{tabular}

${ }^{a}$ TDS: Tobacco Dependence Screener.

${ }^{b}$ FTND: Fagerström Test for Nicotine Dependence.

${ }^{c}$ KTSND: Kano Test for Social Nicotine Dependence.

Table 2. Continuous abstinence rates in percentages from weeks 9-12 (primary outcome) and weeks 9-24 (secondary outcome).

\begin{tabular}{lllll}
\hline Continuous abstinence rate & Telemedicine, mean (SE) & Control, mean (SE) & Difference (95\% CI) & Odds ratio (95\% CI) \\
\hline Weeks 9-12 & $81.0(5.1)$ & $78.9(5.4)$ & $2.1(-12.8$ to 17.0$)$ & $1.14(0.45-2.88)$ \\
Weeks 9-24 & $74.1(5.7)$ & $71.9(6.0)$ & $2.2(-14.0$ to 18.4$)$ & $1.12(0.49-2.57)$ \\
\hline
\end{tabular}

\section{Discussion}

\section{Principal Results}

In this randomized trial, we assessed the efficacy and feasibility of an internet-based remote smoking cessation program compared to the standard face-to-face clinical visit program among patients with nicotine dependence. We found that (1) CARs from weeks 9-12 were relatively high in both groups $(81.0 \%$ in the telemedicine and $78.9 \%$ in the face-to-face control groups) and (2) the clinical efficacy of the telemedicine group was not significantly greater than that of the face-to-face control group in terms of CAR from weeks 9-12.

\section{Comparison with Prior Work}

This trial has several important findings. First, CARs from weeks 9-12 were relatively high. CARs from weeks 9-24 also reached favorable results in both groups $(74.1 \%$ in telemedicine and $71.9 \%$ in control). Compared to other countries [23-25], these CARs estimated using the Japanese smoking cessation program were higher. One of the main reasons could be that all

participants in the Japanese smoking cessation program need to swear to quit smoking and sign a declaration of smoking cessation before participating in the program. This is a unique feature of the Japanese smoking cessation program, and it would strongly contribute to selecting highly motivated participants who want to quit smoking. In terms of previous reports from Japan, Nakamura et al reported CARs from weeks 9-12 and weeks $9-24$ of $65.4 \%$ and $37.7 \%$, respectively, in nicotine-dependent patients receiving $1 \mathrm{mg}$ varenicline on prescription [16]. Japan's Ministry of Health, Labour and Welfare also reported the latest success rate of $63.8 \%$ for smoking cessation at week 12 in nicotine-dependent patients undergoing the standard smoking cessation program [6]. Our trial showed comparable results with these studies, in terms of smoking cessation success rates. In addition, the efficacy of the telemedicine program was not inferior to that of the face-to-face clinic visit program over a prespecified limit value. In summary, telemedicine, or online-based video counseling, could be a viable alternative for managing a smoking cessation program, as it has shown a noninferior efficacy compared to the standard face-to-face clinic visit smoking cessation program. 
Second, the dropout rates of the smoking cessation program were low even at week 24 in both groups, indicating potential improvement in the overall smoking cessation success rates. The Japanese national survey on the efficacy of nicotine-dependence treatment showed a linear relationship between the number of patient visits to outpatient clinics and the treatment success rate [6]. We used the CASC system for both groups in this trial. The system could cover intervals between counseling sessions or clinic visits that help patients obtain clinical guidance. It could also continuously monitor, promote, and encourage commitment to the smoking cessation program [13]. Thus, the CASC system, including the CASC smartphone app, might indirectly contribute to preventing dropouts from both groups.

Third, most of the scores for nicotine dependence in the trial were significantly decreased in both groups. This result indicated that the telemedicine-based and face-to-face smoking cessation programs with the CASC system were effective in improving the status of nicotine dependence and ameliorating craving for smoking over time in the smoking cessation program. However, mood symptoms (depressed, irritable in control, restless, hungry, and poor concentration) of the MPSS did not change during the trial. This outcome may be because the baseline median scores of these symptoms were already low ( $1=$ Not at all or $2=$ Slightly) and continuously stabilized during the trial period.

\section{Strengths and Limitations}

The strength of this trial was that it was the first randomized controlled trial to test the efficacy and feasibility of telemedicine using internet-based video counseling directly compared with face-to-face clinical visit in patients with nicotine dependence. This trial had a few limitations. First, the prespecified limit value of $15 \%$ might not be conservative. However, CARs from weeks 9-12 in both groups were almost what we expected (both around $80 \%$ ), and we considered $65 \%$ of CAR from weeks 9-12 to be a reasonable threshold for checking the clinical relevance of the telemedicine program compared with the face-to-face clinic visit program in accordance with previous reports $[6,16]$. Second, this Web- and smartphone-based program might not be applied for individuals who cannot buy or access mobile devices and did not have enough literacy to read or access the mobile technology. Third, concluding the efficacy of the telemedicine in this 3-months trial could be difficult. Further trials lasting longer than 3 months might be needed to confirm the long-term efficacy of telemedicine. Fourth, we did not collect data on adherence of smoking cessation medications that might affect the trial results.

\section{Conclusions}

Telemedicine using internet-based video counseling for the smoking cessation program had a similar CAR from weeks 9-12 as that of the standard face-to-face clinic visit program. The efficacy of the telemedicine-based smoking cessation program was noninferior to that of the standard clinic visit-based smoking cessation program. The results of this trial demonstrated that internet-based counselling might be a viable alternative to standard clinic visits for smoking cessation.

\section{Acknowledgments}

We thank all the staff and participants involved in this trial. We would also like to express our gratitude to Ayaka Kato for her productive comments on our draft manuscript and Yoshihiko Raita and Masaaki Yamada for their support in conducting the statistical analyses. In addition, we thank Tatsue Takami, Satomi Ono, Naomi Suzuki, Masaru Tukahara and all other administrative and engineering team members in CureApp Inc for their dedicated work for this study.

\section{Conflicts of Interest}

This trial was supported by CureApp, Inc. AN received consulting fees from CureApp, Inc. TT and TM are employees of CureApp, Inc. KS is the founder and a shareholder of CureApp, Inc, and patent holder of the CASC system. EH has a consultation contract as a biostatistician with CureApp, Inc. TO, YF, AK and MM received research support fees from CureApp, Inc.

\section{Multimedia Appendix 1}

Supplemental figures and tables.

[PDF File (Adobe PDF File), 269KB-Multimedia Appendix 1]

\section{Multimedia Appendix 2}

CONSORT-EHEALTH checklist (V 1.6.1).

[PDF File (Adobe PDF File), 3MB-Multimedia Appendix 2]

\section{References}

1. Ikeda N, Inoue M, Iso H, Ikeda S, Satoh T, Noda M, et al. Adult mortality attributable to preventable risk factors for non-communicable diseases and injuries in Japan: a comparative risk assessment. PLoS Med 2012 Jan;9(1):e1001160 [FREE Full text] [doi: 10.1371/journal.pmed.1001160] [Medline: 22291576]

2. World Health Organization. WHO Report on the Global Tobacco Epidemic, 2017 URL: http://apps.who.int/iris/bitstream/ handle/10665/255874/9789241512824-eng.pdf [accessed 2019-04-19] [WebCite Cache ID 77k2EXTmH] 
3. Ikeda N, Saito E, Kondo N, Inoue M, Ikeda S, Satoh T, et al. What has made the population of Japan healthy? Lancet 2011 Sep 17;378(9796):1094-1105. [doi: 10.1016/S0140-6736(11)61055-6] [Medline: 21885105]

4. Igarashi A, Goto R, Suwa K, Yoshikawa R, Ward AJ, Moller J. Cost-Effectiveness Analysis of Smoking Cessation Interventions in Japan Using a Discrete-Event Simulation. Appl Health Econ Health Policy 2016 Feb;14(1):77-87 [FREE Full text] [doi: 10.1007/s40258-015-0204-3] [Medline: 26597111]

5. The Standard Procedure Book for Smoking Cessation, 6th edition (in Japanese). 2014 Apr. URL: https://www.jrs.or.jp/ uploads/uploads/files/information/non-smoking_06.pdf [accessed 2018-12-10] [WebCite Cache ID 74ZVOvjoU]

6. Ministry of Health, Labour and Welfare (Japan). 2017. Report on the Efficacy of Smoking Cessation Treatment Based on Nicotine Dependency Management Fee (in Japanese) URL: http://www.mhlw.go.jp/file/ 05-Shingikai-12404000-Hokenkyoku-Iryouka/0000192293.pdf [accessed 2018-04-29] [WebCite Cache ID 74U5NcjPx]

7. Caponnetto P, Polosa R. Common predictors of smoking cessation in clinical practice. Respir Med 2008 Aug;102(8):1182-1192. [doi: 10.1016/j.rmed.2008.02.017] [Medline: 18586479]

8. Ekeland AG, Bowes A, Flottorp S. Effectiveness of telemedicine: a systematic review of reviews. Int J Med Inform 2010 Nov;79(11):736-771. [doi: 10.1016/j.ijmedinf.2010.08.006] [Medline: 20884286]

9. Taylor GMJ, Dalili MN, Semwal M, Civljak M, Sheikh A, Car J. Internet-based interventions for smoking cessation. Cochrane Database Syst Rev 2017 Sep 04;9:CD007078. [doi: 10.1002/14651858.CD007078.pub5] [Medline: 28869775]

10. Kitada M. Evaluation on the impact of telemedicine for clinic visit for treatment of nicotine dependence and its treatment success. Kinen-Kagaku 2017;11(15):1.

11. Tanigawa T, Nomura A, Kuroda M, Muto T, Hida E, Satake K. A Multi-center, Open-label, Randomized Controlled Trial Comparing Telemedicine and Face-to-Face Consultation Based on the Standard Smoking Cessation Program for Nicotine Dependence: Study Protocol. JMIR Res Protoc (upcoming) 2019:1. [doi: 10.2196/preprints.12701]

12. Ministry of Health, Labour and Welfare (Japan). 2018. Guideline for Online Clinical Practice (in Japanese) URL: https:/ /www.mhlw.go.jp/file/05-Shingikai-10801000-Iseikyoku-Soumuka/0000201789.pdf [accessed 2019-04-19] [WebCite Cache ID 77k3M4hHr]

13. Nomura A, Tateno H, Masaki K, Muto T, Suzuki S, Satake K, et al. A Novel Smoking Cessation Smartphone App Integrated With a Mobile Carbon Monoxide Checker for Smoking Cessation Treatment: Protocol for a Randomized Controlled Trial. JMIR Res Protoc 2019 Feb 11;8(2):e12252 [FREE Full text] [doi: 10.2196/12252] [Medline: $\underline{30741641]}$

14. Kawakami N, Takatsuka N, Inaba S, Shimizu H. Development of a screening questionnaire for tobacco/nicotine dependence according to ICD-10, DSM-III-R, and DSM-IV. Addict Behav 1999;24(2):155-166. [Medline: 10336098]

15. Masaki K, Tateno H, Kameyama N, Morino E, Watanabe R, Sekine K, et al. Impact of a Novel Smartphone App (CureApp Smoking Cessation) on Nicotine Dependence: Prospective Single-Arm Interventional Pilot Study. JMIR Mhealth Uhealth 2019 Feb 19;7(2):e12694. [doi: 10.2196/12694]

16. Nakamura M, Oshima A, Fujimoto Y, Maruyama N, Ishibashi T, Reeves KR. Efficacy and tolerability of varenicline, an alpha4beta2 nicotinic acetylcholine receptor partial agonist, in a 12-week, randomized, placebo-controlled, dose-response study with 40-week follow-up for smoking cessation in Japanese smokers. Clin Ther 2007 Jun;29(6): 1040-1056. [doi: 10.1016/j.clinthera.2007.06.012] [Medline: 17692720]

17. West R, Hajek P. Evaluation of the mood and physical symptoms scale (MPSS) to assess cigarette withdrawal. Psychopharmacology (Berl) 2004 Dec;177(1-2):195-199. [doi: 10.1007/s00213-004-1923-6] [Medline: 15179542]

18. Berlin I, Singleton EG, Heishman SJ. Validity of the 12-item French version of the Tobacco Craving Questionnaire in treatment-seeking smokers. Nicotine Tob Res 2010 May;12(5):500-507 [FREE Full text] [doi: 10.1093/ntr/ntq039] [Medline: 20335281]

19. Otani T, Yoshii C, Kano M, Kitada M, Inagaki K, Kurioka N, et al. Validity and reliability of Kano Test for Social Nicotine Dependence. Ann Epidemiol 2009 Nov;19(11):815-822. [doi: 10.1016/j.annepidem.2009.03.016] [Medline: 19457683]

20. Masaki K, Tateno H, Morino E. A Multi-center, Single-Arm Study to Evaluate Efficacy of Smartphone Application CureApp - for Smoking Cessation: Interim Report. 2017 Jul 09 Presented at: The 634th Kanto Regional Meeting, Japanese Society of Internal Medicine; Sept 07, 2017; Tokyo, Japan.

21. Ministry of Health, Labour and Welfare (Japan). 2008. Report on the Survey of Success Rate of Smoking Cessation Treatment at Health Insurance-Covered Medical Facilities to Reimburse Nicotine Dependency Management Fee (in Japanese) URL: http://www.mhlw.go.jp/shingi/2008/07/dl/s0709-8k.pdf [accessed 2019-11-04] [WebCite Cache ID 74U5umcPo]

22. Masaki K, Tateno H, Kameyama N. A Multi-center, Single-Arm Study to Evaluate Efficacy of Smartphone Application CureApp - for Smoking Cessation. 2018 Apr 14 Presented at: The Japanese Society of Internal Medicine, Annual Scientific Meeting 2018; Apr 14, 2018; Kyoto, Japan.

23. Gonzales D, Rennard SI, Nides M, Oncken C, Azoulay S, Billing CB, Varenicline Phase 3 Study Group. Varenicline, an alpha4beta2 nicotinic acetylcholine receptor partial agonist, vs sustained-release bupropion and placebo for smoking cessation: a randomized controlled trial. JAMA 2006 Jul 05;296(1):47-55. [doi: 10.1001/jama.296.1.47] [Medline: 16820546]

24. Jorenby D, Hays J, Rigotti N, Azoulay S, Watsky E, Williams K, Varenicline Phase 3 Study Group. Efficacy of varenicline, an alpha4beta2 nicotinic acetylcholine receptor partial agonist, vs placebo or sustained-release bupropion for smoking cessation: a randomized controlled trial. JAMA 2006 Jul 05;296(1):56-63. [doi: 10.1001/jama.296.1.56] [Medline: 16820547] 
25. Tonstad S, Tønnesen P, Hajek P, Williams KE, Billing CB, Reeves KR, Varenicline Phase 3 Study Group. Effect of maintenance therapy with varenicline on smoking cessation: a randomized controlled trial. JAMA 2006 Jul 05;296(1):64-71. [doi: $10.1001 /$ jama.296.1.64] [Medline: 16820548 ]

\author{
Abbreviations \\ CAR: continuous abstinence rate \\ CO: carbon monoxide \\ FTCQ-12: 12 -item French version of the Tobacco Craving Questionnaire \\ KTSND: Kano Test for Social Nicotine Dependence \\ MPSS: Mood and Physical Symptoms Scale \\ NDCS: Nicotine Dependence Cognition Scale \\ OR: odds ratio
}

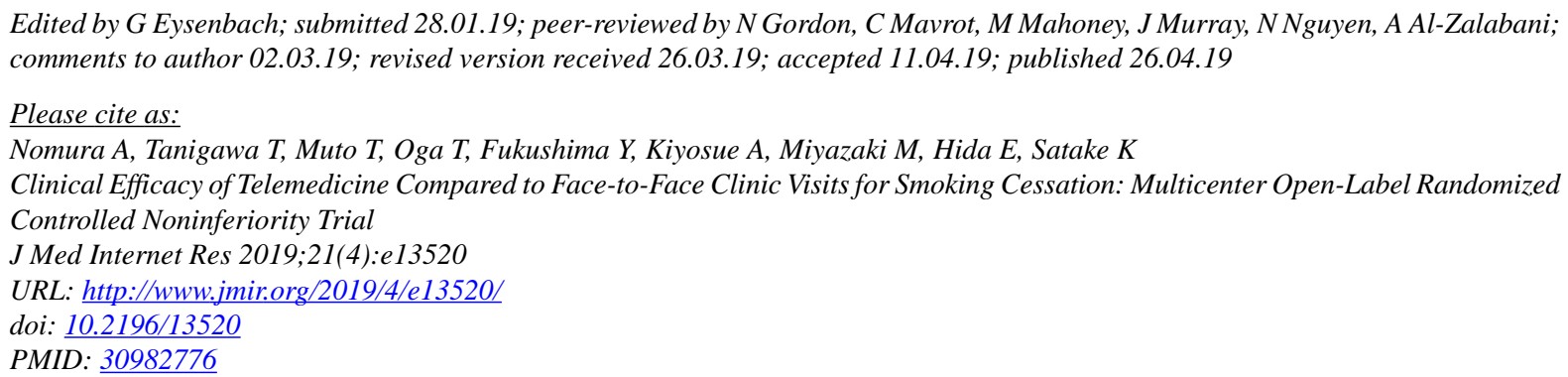

(C)Akihiro Nomura, Tomoyuki Tanigawa, Tomoyasu Muto, Takafumi Oga, Yasushi Fukushima, Arihiro Kiyosue, Masaki Miyazaki, Eisuke Hida, Kohta Satake. Originally published in the Journal of Medical Internet Research (http://www.jmir.org), 26.04.2019. This is an open-access article distributed under the terms of the Creative Commons Attribution License (https://creativecommons.org/licenses/by/4.0/), which permits unrestricted use, distribution, and reproduction in any medium, provided the original work, first published in the Journal of Medical Internet Research, is properly cited. The complete bibliographic information, a link to the original publication on http://www.jmir.org/, as well as this copyright and license information must be included. 